\title{
Cutting the Same Fraction of Several Measures
}

\author{
Arseniy Akopyan • Roman Karasev
}

Received: 4 May 2012 / Revised: 20 July 2012 / Accepted: 24 July 2012 /

Published online: 9 August 2012

(C) Springer Science+Business Media, LLC 2012

\begin{abstract}
We study some measure partition problems: Cut the same positive fraction of $d+1$ measures in $\mathbb{R}^{d}$ with a hyperplane or find a convex subset of $\mathbb{R}^{d}$ on which $d+1$ given measures have the same prescribed value. For both problems positive answers are given under some additional assumptions.
\end{abstract}

Keywords The ham sandwich theorem · Balanced partition

\section{Introduction}

The famous "ham sandwich" theorem of Stone, Tukey, and Steinhaus [13, 14] asserts that every $d$ absolutely continuous probability measures in $\mathbb{R}^{d}$ can be simultaneously partitioned into equal parts by a single hyperplane.

In [3] Bereg and Kano raise the following question (in the planar case): If we are given $d+1$ measures in $\mathbb{R}^{d}$ and want to cut the same (but unknown) fraction of every measure by a hyperplane, then what assumptions on the measures allow us to do so? Certainly, additional assumptions are required because if the measures

\footnotetext{
A. Akopyan ( $₫)$

Institute for Information Transmission Problems RAS, Bolshoy Karetny per. 19, Moscow 127994, Russia

e-mail: akopjan@gmail.com
}

\section{A. Akopyan · R. Karasev}

B.N. Delone International Laboratory "Discrete and Computational Geometry", P.G. Demidov Yaroslavl State University, Sovetskaya st. 14, Yaroslavl' 150000, Russia

R. Karasev

Dept. of Mathematics, Moscow Institute of Physics and Technology, Institutskiy per. 9, Dolgoprudny 141700, Russia

e-mail: r_n_karasev@mail.ru 
are concentrated near vertices of a $d$-simplex then such a fraction cut is impossible. A sufficient assumption is described below.

Definition 1 Let $\mu_{0}, \mu_{1}, \ldots, \mu_{d}$ be absolutely continuous probability measures on $\mathbb{R}^{d}$ and let $\varepsilon \in(0,1 / 2)$. Call the set of measures $\varepsilon$-not-permuted if for any halfspace $H$ the inequalities $\mu_{i}(H)<\varepsilon$ for all $i=0,1, \ldots, d$ imply

$$
\mu_{i}(H) \geq \mu_{j}(H), \quad \text { for some } i<j .
$$

Remark 1 For $\varepsilon>0$ consider all halfspaces $H$ in $\mathbb{R}^{d}$ such that $\mu_{i}(H)<\varepsilon$ for all $i$ and the values $\mu_{i}(H)$ are pairwise distinct. If we arrange the values $\mu_{i}(H)$ in the ascending order, then we get some permutation of $\{0,1, \ldots, d\}$. So there is an order of $\mu_{i}$ such that the measures $\mu_{i}$ are $\varepsilon$-not-permuted if and only if in such a way we cannot get all possible permutations of the $d+1$ element set. In the sequel, for example in Theorem 1, we are actually interested in measures that are $\varepsilon$-not-permuted for at least one order of them.

Remark 2 A natural example of $\varepsilon$-not-permuted measures appears when the support of one measure lies in the interior of the convex hull of the union of supports of the other $d$ measures. In this case the measures are $\varepsilon$-not-permuted for sufficiently small $\varepsilon$.

Now we state the main result.

Theorem 1 Suppose $\mu_{0}, \mu_{1}, \ldots, \mu_{d}$ are absolutely continuous probability $\varepsilon$-notpermuted measures in $\mathbb{R}^{d}$ for some $\varepsilon \in(0,1 / 2)$. Then there exists a halfspace $H$ such that

$$
\mu_{0}(H)=\mu_{1}(H)=\cdots=\mu_{d}(H) \in[\varepsilon, 1 / 2] .
$$

Note that in $[2,6,8]$ a similar problem was considered: Cut by a hyperplane a prescribed fraction of each of $d$ measures in $\mathbb{R}^{d}$. Again, this cannot be done in general and some additional assumptions are needed.

A straightforward consequence of Theorem 1 follows by considering one measure concentrated near a point.

Corollary 1 Suppose $\mu_{1}, \ldots, \mu_{d}$ are absolutely continuous probability measures in $\mathbb{R}^{d}$ and $p$ is a point in the convex hull of their supports. Then there exists a halfspace $H$ such that

$$
\mu_{1}(H)=\mu_{2}(H)=\cdots=\mu_{d}(H)
$$

and $p \in \partial H$.

In Sect. 4 we consider a discrete version of Theorem 1, replacing measures by finite point sets. This is in accordance with the initial statement of the problem in [3].

Finally, in Sect. 5 we consider a problem of cutting the same prescribed fraction of every measure, this time allowing cutting with a convex subset of $\mathbb{R}^{d}$. 


\section{Ham Sandwich Theorem for Signed Measures}

In order to prove Theorem 1 we need a version of the "ham sandwich" theorem [13, 14] for signed measures.

Definition 2 A difference $\rho=\mu^{\prime}-\mu^{\prime \prime}$ of two absolutely continuous finite measures on $\mathbb{R}^{d}$ is called a signed measure. In other words, a signed measure is represented by its density from $L_{1}\left(\mathbb{R}^{d}\right)$.

Theorem 2 (Ham sandwich for signed measures) Suppose we are given $d$ signed measures $\rho_{1}, \ldots, \rho_{d}$ in $\mathbb{R}^{d}$, then there exists a (possibly degenerate) halfspace $H$ such that for any $i$,

$$
\rho_{i}(H)=1 / 2 \rho_{i}\left(\mathbb{R}^{d}\right) .
$$

Remark 3 A degenerate halfspace is either $\emptyset$ or the whole $\mathbb{R}^{d}$. We cannot exclude degenerate halfspaces in this theorem when $\rho_{i}\left(\mathbb{R}^{d}\right)$ 's are all zero. The reason is the same for which we cannot exclude the " $\varepsilon$-not-permuted" assumption from Theorem 1, see also Remark 4 below.

Proof The classical proof from the book of Matoušek [11] passes for signed measures (the authors learned this fact long ago from Vladimir Dol'nikov). Identify $\mathbb{R}^{d}$ with $\mathbb{R}^{d} \times\{1\} \subset \mathbb{R}^{d+1}$. Parameterize halfspaces $\tilde{H} \subset \mathbb{R}^{d+1}$ with boundary passing through the origin by their inner normals. So all halfspaces $H=\tilde{H} \cap \mathbb{R}^{d} \times\{1\}$ (including degenerate) are parameterized by the unit sphere $S^{d}$.

If we map every halfspace to the vector

$$
\left(\rho_{1}(H)-\rho_{1}\left(\mathbb{R}^{d} \backslash H\right), \ldots, \rho_{d}(H)-\rho_{d}\left(\mathbb{R}^{d} \backslash H\right)\right),
$$

then we obtain a continuous odd map $P: S^{d} \rightarrow \mathbb{R}^{d}$; by the Borsuk-Ulam theorem [5] (see also [11]) one halfspace must be mapped to zero.

\section{Proof of Theorem 1}

As the first attempt we try to apply the ham sandwich theorem for signed measures to

$$
\rho_{1}=\mu_{1}-\mu_{0}, \rho_{2}=\mu_{2}-\mu_{1}, \ldots, \rho_{d}=\mu_{d}-\mu_{d-1} .
$$

This way we easily obtain a halfspace $H$ such that $\mu_{0}(H)=\mu_{1}(H)=\cdots=\mu_{d}(H)$. But the halfspace $H$ may be degenerate or $\mu_{i}(H)$ may be all zero. This is not what we want.

Remark 4 By the way, we see that starting from three measures $\mu_{i}$ on $\mathbb{R}^{2}$ distributed along three rays emanating from vertices of a regular triangle $T$ and going in the direction opposite to the center of $T$, we cannot cut the same fraction (possibly zero) of these measures with a non-degenerate halfplane. The same example generalizes to 
higher dimensions and shows that in the ham sandwich theorem for signed measures we cannot avoid using degenerate halfspaces when all measures satisfy $\rho_{i}\left(\mathbb{R}^{d}\right)=0$.

So let us perturb the signed measures with a small positive parameter $s$ :

$$
\rho_{i}^{s}=(1+s) \mu_{i}-\mu_{i-1}, \quad \text { for } i=1, \ldots, d .
$$

Now Theorem 2 gives a halfspace $H$ with:

$$
\rho_{1}^{s}(H)=\cdots=\rho_{d}^{s}(H)=s / 2 .
$$

This is equivalent to the following equalities:

$$
\mu_{i-1}(H)=(1+s) \mu_{i}(H)-s / 2=\mu_{i}(H)+s\left(\mu_{i}(H)-1 / 2\right) .
$$

Suppose $\mu_{i}(H)<\varepsilon$ for all $i=0, \ldots, d$. Then we have $\mu_{i}(H)<1 / 2$ and $s\left(\mu_{i}(H)-\right.$ $1 / 2)<0$. Hence,

$$
\mu_{i-1}(H)<\mu_{i}(H), \quad \text { for } i=1, \ldots, d .
$$

This contradicts the assumption that the measures are $\varepsilon$-not-permuted.

Hence the inequality $\mu_{i}(H) \geq \varepsilon$ for some $i$ is guaranteed while we decrease $s$ to 0 , and in turn it guarantees (remember that we can interchange $H$ and $\mathbb{R}^{d} \backslash H$ !) that $H$ cannot approach degenerate halfspaces $\emptyset$ and $\mathbb{R}^{d}$. So we assume by compactness that $H$ tends to a certain halfspace as $s \rightarrow 0$ and going to the limit in (2) together with (1) we obtain the conclusion.

\section{Discrete Version}

In the paper [3] Bereg and Kano consider a discrete version of this theorem in the plane. They call a line $\ell$ balanced if each halfplane bounded by $\ell$ contains precisely the same number of points of each color.

Theorem 3 (S. Bereg and M. Kano [3]) Let $S$ be a set of $3 n \geq 6$ points in the plane in general position colored in red/blue/green such that

(i) the number of points of each color is $n$;

(ii) the vertices of the convex hull of $S$ have the same color.

Then there exists a balanced line of $S$.

Here we generalize the result of Bereg and Kano. For every directed line $\ell$ we write $x \leq_{\ell} y$ if the projection of $x$ to $\ell$ has not greater coordinate than the projection of $y$ to $\ell$.

Theorem 4 Let $S$ be a set of $(d+1) n$ points in $\mathbb{R}^{d}$ in general position partitioned into colors $S=S_{0} \cup S_{1} \cup \cdots \cup S_{d}$ so that:

(i) the number of points of each $S_{i}$ is $n$; 
(ii) for any directed line $\ell$ there exist two colors $i$ and $j, i<j$, and a point $x \in S_{i}$ such that for any $y \in S_{j}$ we have $y \leq_{\ell} x$.

Then there exists a balanced hyperplane h, or in other words, a hyperplane such that each halfspace bounded by $h$ contains precisely the same number of points of each color.

Proof The proof follows almost directly from the continuous version. Replace each point by a solid ball centered in it and of radius $r>0$ sufficiently small so that there is no hyperplane that intersects any $d+1$ balls at the same time and for any partition of points of $S$ by a hyperplane there exists a hyperplane that separates the corresponding balls accordingly. Since the points are in general position, such $r$ does exist.

Balls of each color generate absolutely continuous measures. So we have $d+1$ absolutely continuous measures; multiplying them by the same constant we make these measures normalized; denote them by $\mu_{0}, \mu_{1}, \ldots, \mu_{d}$.

Now, as in the proof of Theorem 1, we consider signed measures $\rho_{i}^{s}=(1+s) \mu_{i}-$ $\mu_{i-1}$ and find a halfspace $H$ such that $\rho_{1}^{s}(H)=\cdots=\rho_{d}^{s}(H)=s / 2$.

We show that $\mu_{i}(H)$ cannot be less than $1 / n$ for all $i$. Suppose it is so. Then again using (2) we get:

$$
\mu_{i-1}(H)<\mu_{i}(H), \quad \text { for } i=1, \ldots, d .
$$

Consider a line $\ell$ inner-normal to $H$ and colors $i$ and $j$ from condition (ii) for this line. Suppose $\mu_{i}(H)<\mu_{j}(H)<1 / n$. The hyperplane $\partial H$ intersects at least two balls of color $j$, otherwise the inequality would be $\mu_{i}(H) \geq \mu_{j}(H)$ by condition (ii). Under the above assumptions, every hyperplane can pass through at most $d$ balls. Therefore there exist two colors $k$ and $m$ that do not intersect with $\partial H$ and therefore with $H$ (in the opposite case $\mu_{k}(H)$ or $\mu_{m}(H)$ would be at least $1 / n$ ). Hence we have $\mu_{k}(H)=\mu_{m}(H)=0$ in contradiction with (4). So for at least one $i$ the measure $\mu_{i}(H)$ is at least $1 / n$.

As $s$ goes to 0 , the halfspace $H$ tends to a certain halfspace $H_{0}$. Its border hyperplane $h=\partial H$ cuts an equal positive fraction of every measure $\mu_{i}$.

If $h$ touches no ball where the measures are concentrated, then we are done. But a problem can occur if $h$ intersects some balls. Since the points in $S$ are in general position, the hyperplane $h$ touches at most $d$ of them (denote the corresponding subset of $X$ by $I$ ) and we can perturb $h$ so that an arbitrary subset $J \subseteq I$ will be on one side of $h$ while $I \backslash J$ will be on the other side of $h$. Thus we may "round" the fractions of the measure in any way we want and equalize the numbers of points in $H$ for all the colors (compare with the proof of Corollary 3.1.3 in [11]).

Remark 5 As in Remark 1 we can describe point sets satisfying condition (ii) in terms of permutations: For any directed line $\ell$ the order of points with least (among the points of the same color) projection to $\ell$ gives a permutation of colors $\{0,1, \ldots, d\}$; a colored point set satisfies condition (ii) for some order of colors if and only if we cannot get all permutations this way.

Let us give a simpler but still useful assumption on $S$ : 
Corollary 2 Let $S$ be a set of $(d+1) n$ points in $\mathbb{R}^{d}$ in general position colored in colors $0,1, \ldots, d$ so that:

(i) the number of points of each color is $n$;

(ii) points of one color lie in the convex hull of the union of points of the other $d$ colors.

Then there exists a balanced hyperplane $h$.

\section{Cutting a Prescribed Fraction by a Convex Set}

Let us state another problem about cutting the same fraction of several measures:

Problem 1 The dimension $d$ and the number of measures $k>1$ are given. For which $\alpha \in(0,1)$ for any absolutely continuous probability measures $\mu_{1}, \ldots, \mu_{k}$ on $\mathbb{R}^{d}$ it is always possible to find a convex subset $C \subset \mathbb{R}^{d}$ such that

$$
\mu_{1}(C)=\cdots=\mu_{k}(C)=\alpha ?
$$

For $\alpha=1 / 2$ and $k=d$ a positive solution to this problem follows from the ham sandwich theorem. If $\alpha>1 / 2$ then considering two measures, one concentrated near the origin and the other concentrated uniformly near a unit sphere, we see that there is no solution.

If $k>d+1$, one can consider $d+2$ measures concentrated near the vertices of a simplex $S$ and the mass center $w$ of $S$; in this case, any such $C$ must contain a neighborhood of $w$ and therefore cannot cut $\alpha$ of the corresponding measure.

In [15] Stromquist and Woodall solved a similar problem for $k$ measures on a circle and cutting it by a union of $k$ arcs.

Using the results on measure equipartitions from $[1,9,12]$ we are able to solve Problem 1 for $d+1$ measures:

Theorem 5 Suppose $\mu_{0}, \mu_{1}, \ldots, \mu_{d}$ are absolutely continuous probability measures on $\mathbb{R}^{d}$ and $\alpha \in(0,1)$. It is always possible to find a convex subset $C \subset \mathbb{R}^{d}$ such that

$$
\mu_{0}(C)=\mu_{1}(C)=\cdots=\mu_{d}(C)=\alpha,
$$

if and only if $\alpha=1 / m$ for a positive integer $m$.

Proof First, we prove that it is possible if $\alpha=1 / \mathrm{m}$. Following [12] it is sufficient to consider the case when $m$ is a prime and use induction. Consider the space of functions

$$
L=\left\{a_{0}+\sum_{i=1}^{d} a_{i} x_{i}+b \sum_{i=1}^{d} x_{i}^{2}\right\} .
$$

This space has dimension $d+2$. Recall that a generalized Voronoi partition corresponding to an $m$-tuple of pairwise distinct functions $\left\{f_{1}, \ldots, f_{m}\right\} \subset L$ is defined 
by

$$
C_{i}=\left\{x \in \mathbb{R}^{d}: f_{i}(x) \leq f_{j}(x) \text { for all } j \neq i\right\} .
$$

In this case, [9, Theorem 1.3] asserts that there exists a generalized Voronoi partition corresponding to a subset $\left\{f_{1}, \ldots, f_{m}\right\} \subset L$ that equipartitions every $\mu_{i}$. As it was noted by Pablo Soberón, we may not refer to [9, Theorem 1.3] and use the simpler result of [12] if we lift the measures $\mu_{i}$ to the paraboloid $x_{d+1}=x_{1}^{2}+\cdots+x_{d}^{2}$ in $\mathbb{R}^{d+1}$ and then partition them with a convex generalized Voronoi partition.

Assume without loss of generality that $f_{1}(x)$ has the largest coefficient at $\sum_{i=1}^{d} x_{i}^{2}$ among all $f_{j}(x)$. Then the defining equations for $C_{1}$ will look like

$$
\left(b_{1}-b_{j}\right) \sum_{i=1}^{d} x_{i}^{2}+\lambda(x) \leq 0,
$$

where $\lambda(x)$ is a linear function and $b_{1}-b_{j}$ is nonnegative. Note that each of these equations defines either a halfspace or a ball and therefore their intersection $C_{1}$ is convex.

Now we give a counterexample for $d=1$ and $\alpha$ not of the form $1 / m$. Assume $\frac{1}{n}>\alpha>\frac{1}{n+1}$. Let $\mu_{0}$ be the uniform measure on $(0,1)$.

Let $a_{i}, i=1, \ldots, n$, be the points with coordinates $\frac{i}{n+1}$ and $\Delta_{i}$ be the intervals with centers at $a_{i}$ and length $\epsilon<\alpha-\frac{1}{n+1}$. The support of the measure $\mu_{1}$ is the union of intervals $\Delta_{i}$ and $\int_{\Delta_{i}} d \mu_{1}=\frac{1}{n}$ for each $i$ (Fig. 1).

It is easy to see that each convex set $C$ with $\mu_{0}(C)=\alpha$ is an interval of length $\alpha$ in intersection with $(0,1)$ and it must contain at least one interval $\Delta_{i}$. Therefore $\mu_{1}(C) \geq \frac{1}{n}>\alpha$.

For $d>1$ we can extend the one-dimensional example. Consider a $d$-dimensional regular simplex with vertices $v_{0}, v_{1}, \ldots, v_{d}$. Let the measures $\mu_{2}, \ldots, \mu_{d}$ concentrate near the vertices $v_{2}, \ldots, v_{d}$, respectively. Similarly to the case $d=1$, let $\mu_{0}$ be the uniform measure on a small cylinder around the edge $v_{0} v_{1}$. The measure $\mu_{1}$ will look like in the one-dimensional case, but its support will be intervals on the segment that connects the centers of faces $v_{0} v_{2} \ldots v_{d}$ and $v_{1} v_{2} \ldots v_{d}$. See Fig. 2 for the twodimensional case.

Fig. 1 Two measures in one dimension

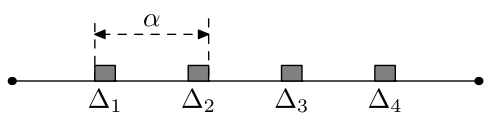

Fig. 2 Three measures in two dimensions

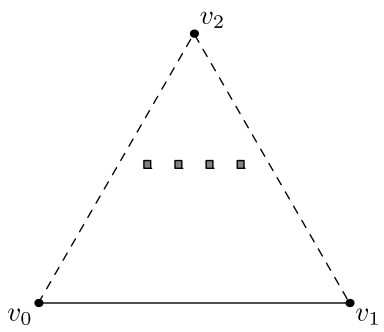


To conclude the proof we note the following. Assume that the measures $\mu_{i}$ are concentrated in $\delta$-neighborhoods of their respective vertices (for $i>1$ ) or segments (for $i=0,1$ ). Then going to the limit $\delta \rightarrow+0$ and using the Blaschke selection theorem, we assume that the corresponding $C_{\delta}$ tends in the Hausdorff metric to some $C_{0}$. This $C_{0}$ must intersect the segment $\left[v_{0}, v_{1}\right]$ by an interval of length at least $\alpha\left|v_{0}-v_{1}\right|$. It also has to contain every vertex $v_{i}$ for $i=2, \ldots, d$. Hence, similarly to the onedimensional case, $C_{0}$ contains in its interior one of the support segments of $\mu_{1}$. For small enough $\delta$, the convex set $C_{\delta}$ will also contain at least $1 / n$ of the measure $\mu_{1}$, which is a contradiction.

Remark 6 For $d=1$ this result follows from the theorem of Levy [10] about a segment on a curve. Note that Hopf [7] showed that the set of $\alpha$ for which the required segment does not exist is additive.

Remark 7 In [4, Theorem 3.2] it was proved that any two absolutely continuous probability measures on $S^{2}$ can be cut into pieces of measures $\alpha, \alpha, 1-2 \alpha$ by a 3 -fan. Using the central projection we see that any two absolutely continuous probability measures on $\mathbb{R}^{2}$ may be cut into pieces of measures $\alpha, \alpha, 1-2 \alpha$ with a (possibly degenerate) 3-fan. It is clear that at least one of the $\alpha$ parts of the fan is a convex angle and therefore Problem 1 has a positive solution for $k=d=2$ and any $\alpha \in(0,1 / 2]$.

Acknowledgements We thank Nikolay Dolbilin for drawing our attention to this problem. We also thank Fedor Petrov, Imre Bárány, Pavle Blagojević, Pablo Soberón and two unknown referees for useful remarks.

The research of both authors is supported by the Dynasty Foundation, the President's of Russian Federation grant MD-352.2012.1, the Russian Foundation for Basic Research grant 10-01-00096, the Federal Program "Scientific and Scientific-Pedagogical Staff of Innovative Russia" 2009-2013, and the Russian Government Project 11.G34.31.0053. The research of A.V. Akopyan is also supported by the Russian Foundation for Basic Research grant 11-01-00735, and the research of R.N. Karasev is also supported by the Russian Foundation for Basic Research grant 10-01-00139.

\section{References}

1. Aronov, B., Hubard, A.: Convex equipartitions of volume and surface area. arXiv preprint: arXiv:1010.4611 (2010)

2. Bárány, I., Hubard, A., Jerónimo, J.: Slicing convex sets and measures by a hyperplane. Discrete Comput. Geom. 39, 67-75 (2008). 10.1007/s00454-007-9021-2

3. Bereg, S., Kano, M.: Balanced line for a 3-colored point set in the plane. Electron. J. Comb. 19(1), P33 (2012)

4. Blagojević, P.V.M., Blagojević, A.S.D.: Using equivariant obstruction theory in combinatorial geometry. Topol. Appl. 154(14), 2635-2655 (2007). doi:10.1016/j.topol.2007.04.007

5. Borsuk, K.: Drei sätze über die $n$-dimensionale euklidische Sphäre. Fundam. Math. 20(1), 177-190 (1933)

6. Breuer, F.: Uneven splitting of ham sandwiches. Discrete Comput. Geom. 43(4), 876-892 (2010)

7. Hopf, H.: Über die Sehnen ebener Kontinuen und die Schleifen geschlossener Wege. Comment. Math. Helv. 9, 303-319 (1936). 10.1007/BF01258195

8. Karasev, R.N.: Theorems of Borsuk-Ulam type for flats and common transversals of families of convex compact sets. Sb. Math. 200(10), 1453-1471 (2009). doi:10.1070/ SM2009v200n10ABEH004045

9. Karasev, R.N.: Equipartition of several measures. arXiv preprint: arXiv:1011.4762 (2010)

10. Levy, P.: Sur une généralisation du théorème de Rolle. C. R. Acad. Sci., Paris 198, 424-425 (1934) 
11. Matoušek, J.: Using the Borsuk-Ulam Theorem: Lectures on Topological Methods in Combinatorics and Geometry. Springer, Berlin (2003)

12. Soberón, P.: Balanced convex partitions of measures in $\mathbb{R}^{d}$. Mathematika 58(1), 71-76 (2012). doi:10.1112/S0025579311001914

13. Steinhaus, H.: Sur la division des ensembles de l'espace par les plans et des ensembles plans par les cercles. Fundam. Math. 33, 245-263 (1945)

14. Stone, A.H., Tukey, J.W.: Generalized "sandwich" theorems. Duke Math. J. 9(2), 356-359 (1942)

15. Stromquist, W., Woodall, D.: Sets on which several measures agree. J. Math. Anal. Appl. 108, 241248 (1985). doi:10.1016/0022-247X(85)90021-6 